\title{
Mouse models uncap novel roles of SSBs
}

\author{
Cell Research (2013) 23:744-745. doi:10.1038/cr.2013.63; published online 7 May 2013
}

Three recently published reports, including one in Cell Research, generated Ssb1 knockout mice and demonstrated critical roles of this protein in regulating skeletogenesis, telomere homeostasis and tumor suppression.

Single-stranded DNA binding proteins (SSBs) regulate numerous cellular transactions and are typified by the presence of conserved oligosaccharideoligonucleotide (OB)-fold binding domains. RPA, the canonical SSB in eukaryotes, is an essential component of DNA replication and repair. Recently, our group discovered that the human genome encoded two additional SSB proteins, hSSB1 and hSSB2, which show greater sequence and domain homology to the archaeal and bacterial SSBs than RPA [1]. Functional evaluation of hSSB1 using siRNAbased depletion in human cells showed severe perturbation of ATM kinase activation in response to ionizing radiation, diminished DNA DSB repair by homologous recombination, and general genomic instability [1]. More recently, the repair roles of hSSB1 and hSSB2 were shown to depend on their heterotrimerization with two proteins, C9Orf80 and INTS3, a protein originally identified as a component of the Integrator complex that processes snRNA [2-5].

Recently, three laboratories independently generated $S s b 1$ knockout mice, and showed that ablation of Ssb1 leads to perinatal lethality [6-8]. Surprisingly, these reports demonstrated a critical role of Ssb1 in mediating skeletogenesis. The functional cause of perinatal lethality in $S s b 1^{-/-}$newborn pups was largely attributed to severe mispatterning of the dorsal rib-cage resulting in rapid asphyxiation at birth.

Sandy Chang's laboratory assigned a new function to Ssb1 and Ssb2 in mediating telomere homeostasis [7]. Telomeres consist of TTAGGG repeats terminating in a G-rich $3^{\prime}$ single-stranded DNA (ssDNA) overhang, which is usually folded into the duplex DNA to protect it from activating the DNA damage response (DDR). This protection is conferred by a multi-protein complex called Shelterin [9]. Despite a common functionality of the Shelterin complex in protecting telomeres from activating the DDR, individual components of this complex have been shown to elicit specific DDRs upon their removal. For example, the Shelterin component TRF 2 binds to the doublestranded portion of the telomere to suppress the recognition of telomeric ends as DSBs, and prevents ATMdependent canonical non-homologous end joining (c-NHEJ)-mediated chromosomal fusions at the telomeres. In contrast, the TPP1-POT1 (POT1a/b in mouse) components of Shelterin bind the G-rich ssDNA overhang. Removal of POT1a results in ATR-dependent checkpoint activation through CHK1, and cells that escape this checkpoint exhibit chromosomal fusions by alternative-NHEJ (a-NHEJ). Moreover, TPP1-POT1 protects newly replicated telomere ends from post-replicative repair, illustrated by chromatid fusions upon removal of TPP1-POT1 [9]. Given the end-protection conferred by Shelterin, the components of this complex can be manipulated to uncap the telomeres to reveal additional factors involved in telomeric end protection.

Notably, a number of ssDNA binding proteins have been implicated in mediating telomere homeostasis. The
ssDNA binding protein STN1 forms part of the CST complex, which protects the $3^{\prime}$ overhang of telomeres and plays a role in telomere replication [9]. In addition, the shelterin components POT $1 \mathrm{a} / \mathrm{b}$ are ssDNA binding proteins that protect the G-rich overhang in telomeres. Gu et al. [7] investigated the putative role of Ssb1 and Ssb2 in mediating telomere homeostasis utilizing MEFs. Both Ssb1 and Ssb2 localized to a subset of telomeres and a number of telomere-related aberrations were observed in $\mathrm{Ssb1}^{-/-}$MEFs. The authors also showed functional interactions between Ssb1 and POT1a and the binding of POT1a to telomeres was shown to promote Ssb1 accumulation at these sites, suggesting that the TPP1-POT1a heterodimer may function in recruiting Ssb1 to telomeres to mediate their protection. Interestingly, uncapping of telomeres through the removal of TRF2 resulted in a decrease in the proportions of chromosomal fusions in $S s b 1^{-/-}$MEFs, suggesting that Ssb1 promotes ATM-dependent c-NHEJ repair of uncapped telomeres. Conversely, expression of dominant-negative TPP1 in an $S s b 1^{-/}$background led to an increase in a-NHEJ fusions and attenuated CHK1 activation, also implicating Ssb1 in ATR activation at uncapped telomeres. In addition, an increase in chromatid fusions in $S s b 1^{-/-}$ MEFs following dominant-negative TPP1 expression suggests that Ssb1 protects newly replicated telomeres from post-replicative repair. Although the roles of Ssb1 in ATM and ATR activation are in agreement with previously described functions of Ssb1 in human cells [2-4], they contradict the recent reports by Feldhahn et al. [6] and Shi et al. [8] utilizing $S s b 1^{-/}$ 


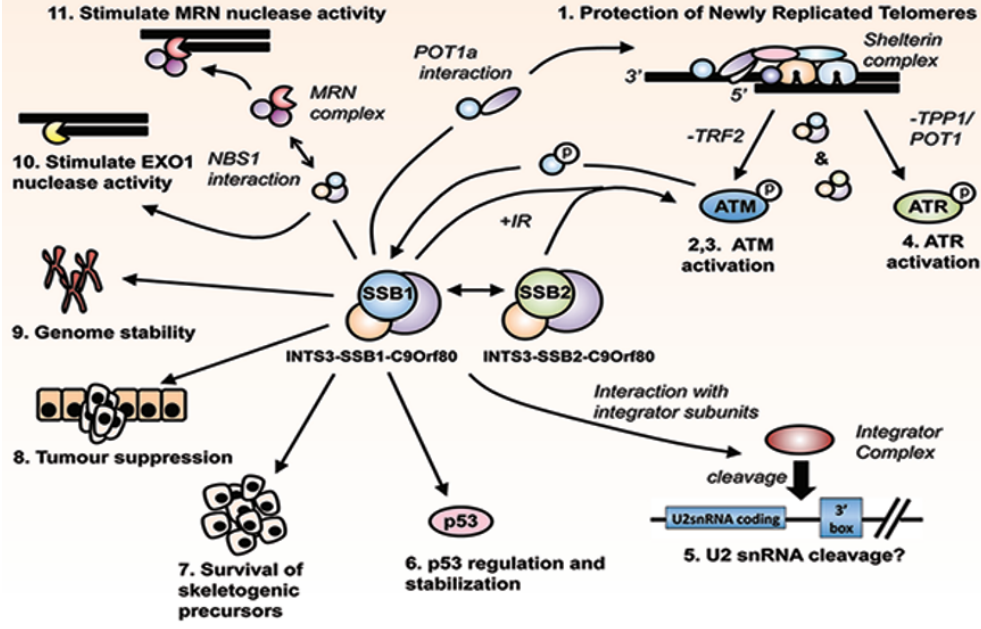

Figure 1 Roles of SSB1/SSB2 and their associated complexes. (1) Ssb1 interacts with Pot1a to associate with telomeres [7]. (2) Ssb1/2 promotes ATM activation in the absence of TRF2 [7]. (3) ATM phosphorylates SSB1 following ionizing radiation to stabilize SSB1 [1]. In turn, SSB1/2 are required for ATM activation [1, 3, 4]. (4) SSB1/2 activates ATR signalling after removal of TPP1/POT1 [7]. (5) SSB1/2 interacts with multiple integrator components, but it remains unclear whether it is involved in canonical integrator activity [10]. (6) SSB1 stabilizes p53 and regulates its transcriptional activity [11]. (7) SSB1 promotes survival of skeletogenic precursors [6, 8]. (8) SSB1 prevents tumorigenesis [8] and (9) promotes genomic stability [1, 7, 8]. (10) INTS3-SSB1-C9Orf80 complexes stimulate EXO1 nuclease activity in vitro [12]. (11) SSB1/INTS3 associates with NBS1 and SSB1 stimulates MRN nuclease activity $[2,13]$.

MEFs. However, the fact that Gu et al. [7] used SV40T immortalized MEFs and the other two studies $[6,8]$ used primary MEFs may provide a context in which these phenotypes could be more readily observed.

Strikingly, all three studies reported pronounced upregulation of $\mathrm{Ssb} 2$ in response to Ssb1 deletion in both $S s b 1^{--}$ tissues and MEFs [6-8]. Given that Ssb2 can substitute for Ssb1 in INTS3SSB-C9Orf80 complexes, these results suggest that Ssb2 may compensate for some roles of Ssb1. However, attempts to deplete Ssb2 even in wild-type MEFs led to rapid cell death, indicating that Ssb2 may play additional roles critical for cell viability $[6,7]$. The study by Gu et al. [7] elegantly circumvented these effects through reconstitution of $S s b 1^{-/-}$MEFs with a mutant Ssb1 construct that does not result in concomitant upregulation of Ssb2. Importantly, reconstitution of $\mathrm{Ssb1}^{-/}$MEFs with this construct caused more pronounced
ATM and ATR signaling deficiencies than deletion of Ssb1 alone, further cementing the role of these proteins in the activation of these pathways.

Despite the partially redundant roles of Ssb1 and Ssb2 described by Gu et al. [7], evidence from the three publications of $\mathrm{Ssbl}^{-/-}$mice suggested that $\mathrm{Ssb} 1$ is required for some aspects of genomic stability in a physiological context. Shi et al. [8] generated tamoxifen-inducible $\mathrm{Ssb1}^{-/}$mice to study the effects of Ssb1 depletion in adult mice. These mice exhibited impaired fertility and diminished testis size. Moreover, induced $\mathrm{Ssb1}^{-/-}$mice were radiosensitive in response to total body irradiation. These mice exhibited broad-spectrum spontaneous tumor formation further supported by $\mathrm{Gu}$ et al. [7] who generated Ssb1 hypomorphic floxed mice that were viable but had a limited lifespan and exhibited increased tumor dissemination on a $p 53^{-/-}$background. These results demonstrate a critical role of Ssb1 in preventing chromosomal instability and cancer progression.

Together, the three reports [6-8] illustrated novel and diverse roles of Ssb1 in mediating skeletogenesis, telomeric homeostasis and genomic stability (Figure 1). Our understanding of the mechanism by which Ssb1 ablation might regulate such diverse phenotypes in mice will require further investigation of its functional relationship with the integrator complex. Given the intimate association of the integrator complex with RNA pol II and the interaction of SSB1 with multiple integrator components [10], it will be interesting to determine whether association of SSBs with integrator components can regulate additional processes within the cell.

Amanda L Bain ${ }^{1}$, Wei Shi ${ }^{1}$, Kum Kum Khanna ${ }^{1}$

${ }^{I}$ Queensland Institute of Medical Research, Herston, Australia

Correspondence: Kum Kum Khanna

E-mail: KumKum.Khanna@qimr.edu.au

\section{References}

1 Richard DJ, Bolderson E, Cubeddu L, et al. Nature 2008; 453:677-681.

2 Huang J, Gong Z, Ghosal G, et al. Mol Cell 2009; 35:384-393.

3 Li Y, Bolderson E, Kumar R, et al. J Biol Chem 2009; 284:23525-23531.

4 Skaar JR, Richard DJ, Saraf A, et al. J Cell Biol 2009; 187:25-32.

5 Zhang F, Wu J, Yu X. J Biol Chem 2009; 284:30408-30415.

6 Feldhahn N, Ferretti E, Robbiani DF, et al. EMBO J 2012; 31:4045-4056.

7 Gu P, Deng W, Lei M, et al. Cell Res 2013; 23:705-719.

8 Shi W, Bain AL, Schwer B, et al. PLoS Genet 2013; 9:e1003298.

9 Stewart JA, Chaiken MF, Wang F, et al. Mutat Res 2012; 730:12-19.

10 Malovannaya A, Li Y, Bulynko Y, et al. Proc Natl Acad Sci USA 2010; 107:2431-2436.

11 Xu S, Wu Y, Chen Q, et al. Cell Res 2013; 23:423-435.

12 Yang SH, Zhou R, Campbell J, et al. EMBO $J$ 2013; 32:126-139.

13 Richard DJ, Cubeddu L, Urquhart AJ, et al. Nucleic Acids Res 2011; 39:3643-3651. 